\title{
Adaptación y validación de la Versión Abreviada de la Escala de Autoritarismos de Derechas (RWA) en una muestra chilena
}

\author{
Adaptation and Validation of the short version of \\ the Right-Wing Authoritarianism Scale (RWA) in a \\ Chilean sample
}

Manuel Cárdenas, Luis Parra

Resumen

Este estudio presenta una adaptación de una versión reducida de la escala de autoritarismo de derechas (RWA). Participaron en el estudio dos muestras de estudiantes secundarios y universitarios. La escala adaptada posee ítems más cortos, con menos palabras extremas en su redacción y menos referencias a grupos específicos. La confiabilidad fue .72 (alpha de Cronbach). Los análisis de regresión múltiple revelaron que la escala de Orientación a la Dominancia Social (SDO) y la escala de nacionalismo contribuyen sustancialmente a la varianza de la escala RWA. La versión corta de la escala RWA parece poseer la estructura de tres dimensiones, predicha por la teoría, mostrando buenos índices de ajuste en el análisis factorial de tipo confirmatorio.

Palabras Clave: Autoritarismo de derechas, Confiabilidad, Validez, Chile.

1 Escuela de Psicología, Universidad Católica del Norte. Chile.

E-mail: jocarde@ucn.cl y lpd001@ucn.cl 


\section{Abstract}

This study presents an adaptation of a short version of the rightwing authoritarianism scale (RWA). Participants were two samples of high school and university students. The scale adapted had items that were shorter, with less extreme wording and less reference to specific groups. The scale reliabilities was .72 (Cronbach's alpha). Multiple regression analyses revealed that the social dominance orientation scale (SDO) and nationalism scale substantially contribute to variance in RWA. The short version of RWA scale seems to possess the structure of three dimensions predicted by the theory, showing proper fit index in the confirmatory factor analysis.

Key words: Right-wing Authoritarianism, Reliability, Validity, Chile.

\section{Introducción}

La escala de autoritarismo de derechas (RWA) fue desarrollada para medir la covariación de tres grupos de actitudes: sumisión autoritaria, agresión autoritaria y convencionalismo (Altemeyer, 1981; 1996). La sumisión refiere al grado de sometimiento a las autoridades percibidas como legítimas dentro de la sociedad en que uno vive. La agresión autoritaria aludiría a una forma de agresividad general, dirigida contra las personas que se perciben como sancionadas por la autoridad legítima. Finalmente, el convencionalismo refiere al grado de adhesión a las normas sociales aprobadas por la sociedad y sus autoridades. En conjunto, estas tres dimensiones apuntarían a saturar el constructo autoritarismo.

El autoritarismo de derechas no es utilizado aquí en sentido de la orientación política (partidos a los que adscribe) o económica (adscripción a un determinado modelo económico o pertenencia a un determinado nivel socioeconómico), sino en un sentido psicológico y social. Este 
autoritarismo es de derechas en el sentido de que se trata de una sumisión a las autoridades establecidas, su objetivo es preservar el orden establecido, agrediendo a aquellos que lo desafían. De esta forma, es muy posible que existan personas autoritarias de derecha aun adscribiendo a ideas políticas de izquierda. Esta conceptualización del autoritarismo de derechas se fundamenta en la asunción de los tres grupos de actitudes definidos anteriormente.

La escala original estaba compuesta por treinta ítems y ha sido utilizada a través de diversas investigaciones en diferentes países (Ahrens \& Innes, 1994; Altemeyer, 1998; Duckitt, 2000; McFarland, Ageyev \& Djintcharadze, 1996; Farre \& Duckitt, 1994, Sidanius \& Pratto, 1999; Ekehammar, Akrami, Gylje \& Zakrisson, 2004; Roccato, Re \& Sclauzero, 2002; Seoane \& Garzón, 1992). También existe una serie de versiones abreviadas de la RWA (Schultz \& Stone, 1994; Walter, 1996; Zakrisson, 2005) que han intentado subsanar algunos problemas de la escala completa, los que refieren básicamente a sus ítems demasiado largos y que contendrían más de una sentencia en su estructura. Lo anterior haría sus contenidos ambiguos, lo que sumado al uso de palabras extremas, de marcado uso cultural (idiosincrásico) y, por ello, sumamente dependiente del contexto, atentaría contra su validez (Schaffer \& Riordan, 2003). Adicionalmente, la versión original de la RWA resulta poco útil en investigación, dado su extensión y el largo tiempo que lleva completarla (y en referencia a la necesidad de utilizar en nuestros estudios otras medidas adicionales), así como los efectos del paso del tiempo que ha puesto en desuso algunos de los términos o palabras empleados en sus reactivos.

La RWA ha mostrado una estrecha relación con una serie de variables psicosociales, entre las que cabe señalar: conservadurismo (Ray, 1985); intolerancia (Duckitt \& Farre, 1994; Thomsen, Green \& Sidanius, 2008); normatividad (Stone \& Schaffner, 1997); obediencia a la autoridad (Altemeyer, 1981); otras medidas de autoritarismo (Meloen, VanderLinden \& De Witte, 1996); y fundamentalismo religioso (Rosenberg, 2006). Adicionalmente, las personas que puntúan alto en la escala RWA tienden a manifestar una serie de actitudes o conductas específicas, entre las que cabe 
mencionar: aceptación y justificación de las injusticias del gobierno (tales como las escuchas telefónicas ilegales y el acoso policial); preferencia por debilitar las garantías constitucionales y limitar las libertades individuales; exigir castigos más duros contra los criminales comunes; ser prejuicioso contra las minorías étnicas o sexuales (Haddock, Zanna \& Esses, 1993; Stones, 2006); insistencia en la asunción de roles sexuales tradicionales, preferencia por "líderes fuertes" e identificación con partidos de derecha; así como presencia de altos niveles de dogmatismo y etnocentrismo (Altemeyer, 1981; 1993; Duckitt, 1992; Roccato, 1997; Rosenberg, 2006).

Por otra parte, se ha señalado en la literatura la estrecha vinculación entre autoritarismo y nacionalismo (Altemeyer, 1993), entendido este último como una forma de obediencia acrítica y una idealización del endogrupo. Es por ello que se ha reportado una baja relación entre autoritarismo y patriotismo (Roccato, Re \& Sclauzero, 2002), donde patriotismo no referiría sino a una fuerte vinculación afectiva con la propia nación (Kosterman \& Feshbach, 1989), de la que no se deduciría desprecio por otros países ni prejuicio hacia los extranjeros (Kellas, 1991). Del mismo modo, se ha reportado un relación alta y consistente entre autoritarismo y dominancia social (Cohrs, Moschner, Maes \& Kielmann, 2005; Dru, 2007; Cohrs \& Abrock, 2009; Cárdenas, Meza, Yáñez \& Lagues, 2010).

La escala RWA ha mostrado índices de consistencia interna adecuados. De este modo, Altemeyer (1981) reporta un alpha de Cronbach de .88 en su muestra original de 956 estudiantes. Otros estudios con muestras de conveniencia reportan coeficientes de fiabilidad de entre .77 y .95 (Zwillenberg, 1983). Para otros contextos nacionales (Australia y Rusia) se reportan coeficientes de .84 y .92, respectivamente (Ray, 1985; McFarland, Agayev \& Abalakina, 1992). La fiabilidad test-retest es reportada en .95 en el periodo de una semana y de .85 para el intervalo de 28 semanas (Altemeyer, 1988; Knight, 1999).

El objetivo del presente estudio será adaptar y validar la versión abreviada de la escala RWA (Zakrisson, 2005), intentando subsanar los problemas detectados en la versión original de la escala RWA. 


\section{Método}

Participantes

El número de participantes para el proceso de adaptación y pilotaje de la escala fue de 117 personas, 52 mujeres (44\%) y 65 hombres (56\%), todos ellos estudiantes de último año de secundaria y cuyas edades fluctuaban entre 16 y 20 años $(\mathrm{M}=16.78$; DT = .99).

Para la validación de la escala nuestra muestra definitiva quedó compuesta por 264 participantes, 151 mujeres (57\%) y 112 hombres (43\%), cuyas edades fluctuaban entre 16 y 33 años $(\mathrm{M}=18.7$; DT = 2.51). Los participantes fueron reclutados entre estudiantes de último año de secundaria y de primer año de universidad, en diferentes cursos, de las carreras de Psicología, Periodismo y Derecho, todos ellos de la ciudad de Antofagasta (Chile). El muestreo de conveniencia fue dirigido de modo incidental, ya que los estudiantes universitarios fueron seleccionados en carreras donde se tenía algún vínculo de docencia o investigación.

\section{Procedimiento}

Los participantes fueron reclutados como voluntarios y contestaron anónimamente el cuestionario de lápiz y papel, en el horario de clases, de modo que la aplicación fue grupal. El tiempo de respuesta aproximado para el cuestionario fue de 20-30 minutos. Los participantes fueron informados de los fines de la investigación y de las condiciones de anonimato y confidencialidad con que se manejarían los datos, los cuales sólo pueden ser usados para fines de investigación. Se siguieron los criterios éticos exigidos por CONICYT y el comité de ética de la Universidad Católica del Norte.

\section{Medidas}

El cuestionario quedó compuesto por dos secciones. En la primera se consultaba a los participantes por algunas variables demográficas y 
psicosociales que pudiesen resultar de interés para este estudio: sexo, edad, posicionamiento político (Ej. "En asuntos políticos frecuentemente de izquierda y derecha ¿dónde situarías tus ideas en la siguiente escala, independientemente de que te intereses o no por la política?". Se entregan siete opciones de respuesta, desde "extrema izquierda" hasta "extrema derecha”) y nivel socioeconómico familiar (ha sido medido por medio del indicador ESOMAR, el cual cruza las variables nivel educativo del principal sostenedor del hogar y categoría ocupacional del principal sostenedor del hogar). En la segunda sección se presentó la escala RWA junto a aquellas medidas que nos servirán como medidas de validez convergente. Las escalas utilizadas fueron las siguientes:

\section{Escala Abreviada de Autoritarismo de Derechas (RWA)}

La escala que hemos utilizado contiene 15 ítems (Zakrisson, 2005), los que fueron traducidos del inglés y adaptados para su uso en la muestra nacional. Esta versión se diferencia de la original (Altemeyer, 1998) en: (a) que los ítems son, generalmente, más cortos; (b) en algunos casos se sustituye la palabra "País" por el término "Sociedad", de modo de reducir el riego de confusión de los ítems con los contenidos de la escala de Nacionalismo; (c) algunas palabras fueron modificadas para quitarles su contenido extremo y hacerlas menos reactivas; (d) se alteraron algunos términos poco usuales en nuestro contexto, los cuales fueron reemplazados por otros de uso más común y más directamente comprensibles para nuestra muestra. Los ítems de la escala tomaron valores entre 1 ("Totalmente en desacuerdo") y 7 ("Totalmente de acuerdo"). Valores altos en la escala indicarían un alto grado de autoritarismo. (Ver escala definitiva en Apéndice: Escala RWA - versión de 12 ítems).

\section{Escala de Orientación a la Dominancia Social (SDO)}

Se trata de la versión de 16 ítems (Pratto, Sidanius, Stallworth \& Malle, 1994; Sidanius \& Pratto, 1999) adaptada para muestra nacional (Cárdenas et al., 2010). La escala fue balanceada, es decir, presenta ocho ítems 
en sentido directo y ocho en sentido inverso. Toma la forma de una escala Likert, que va desde 1 ("Totalmente en desacuerdo") hasta 7 ("Totalmente de acuerdo"), y donde valores elevados indican un alto grado de Dominancia Social. La confiabilidad fue de .77 (coeficiente alpha de Cronbach).

\section{Escala de Nacionalismo (EN)}

Escala de siete ítems, construida por Therune (1964) y validada para muestra nacional (Calderón, 2009). Las puntuaciones para los ítems de la escala toman valores entre 1 ("Totalmente en desacuerdo") y 7 ("Totalmente de acuerdo"). La confiabilidad para la EN fue de .78 (coeficiente alpha de Cronbach). Puntuaciones elevadas indican Nacionalismo.

\section{Escala de Patriotismo (EP)}

Compuesta por diez ítems con puntuaciones que oscilan entre 1 (“Totalmente en desacuerdo") y 7 (“Totalmente de acuerdo"). Esta escala fue construida por Therune (1964) y se encuentra validada para su uso nacional (Calderón, 2009). Puntuaciones altas indican una valoración exacerbada de los valores patrios. La escala ha mostrado ser consistente internamente, obteniendo medidas de confiabilidad para este estudio de .86 (coeficiente alpha de Cronbach).

\section{Escala de Religiosidad (ER).}

Consta de cuatro ítems que indicarían el grado de importancia que el sujeto concede a sus ideas religiosas. La escala toma valores entre 1 ("Totalmente en desacuerdo") y 7 ("Totalmente de acuerdo"). Valores elevados indican que se concede una alta importancia a las propias ideas religiosas en la vida cotidiana. La confiabilidad para la ER fue de .92 (coeficiente alpha de Cronbach). 


\section{Resultados}

\section{Adaptación de la Escala}

La escala RWA fue traducida del inglés al español por dos traductores independientes, utilizando la versión de Zakrisson (2005). Los desacuerdos fueron resueltos mediante la discusión de los traductores y el equipo de investigación. Para la versión utilizada en la etapa de pilotaje se reemplazaron algunos conceptos poco usuales en nuestro contexto por otras palabras que fueran directamente comprensibles por los participantes de nuestra muestra.

\section{Pilotaje y modificaciones}

El análisis de la consistencia interna mostró una fiabilidad de .70 (alpha de Cronbach). El pilotaje nos revela que hay tres ítems que obtienen muy bajas correlaciones con el total. Al revisar su redacción hemos decidido no incluirlos en la versión definitiva de la escala, la que obtiene un coeficiente de confiabilidad de .72, pudiendo alcanzar de este modo una mayor independencia con la escala SDO (ya que eran ítems que aumentaban la correlación entre estas escalas).

\section{Indicaciones de Validez}

Los resultados obtenidos por medio de un análisis factorial (procedimiento de extracción de componentes principales con rotación VARIMAX) nos revelan la presencia de tres factores principales que explican el 50.75\% de la varianza total (índices de adecuación muestral $\mathrm{KMO}=.76 \mathrm{y} \mathrm{X}_{(66)}^{2}=$ 555.06; $\mathrm{p}$ <.001). El primer factor, que explica el $20.75 \%$ de la varianza, queda conformado por los ítems 3, 5, 9, 11 y 12. Estos ítems harían alusión a la dimensión "agresión autoritaria" y apuntarían a una forma de agresividad dirigida contra aquellas personas sancionadas por la autoridad legítima.

El segundo factor, que explica el $17.05 \%$ de la varianza, incluye los ítems 1, 2, 4 y 6. Este conjunto de reactivos apunta a la dimensión "sumisión autoritaria", que contribuye a medir el grado de sumisión de los sujetos a las autoridades legítimas (ver Tabla 1). 
Tabla 1. Cargas factoriales de los items de la RWA

\begin{tabular}{|lccc|}
\hline & Factor I & Factor II & Factor III \\
\hline Ítem 1 & & .53 & \\
Ítem 2 & & .81 & \\
Ítem 3 & .65 & .67 & \\
Ítem 4 & & .62 & \\
Ítem 5 & .69 & & .77 \\
Ítem 6 & & & .60 \\
Ítem 7 & & & \\
Ítem 8 & & .34 & \\
Ítem 9 & .69 & & \\
Ítem 10 & & & \\
Ítem 11 & & & \\
Ítem 12 & .55 & & \\
\hline
\end{tabular}

Finalmente, el tercer factor explica el $12.94 \%$ de la varianza total e incluye los ítems 7, 8 y 10 . Este factor sería equivalente al factor convencionalismo e indicaría un alto grado de adhesión a las normas sociales aprobadas por la autoridad legítima.

Este modelo de tres factores fue testeado por medio de un análisis factorial de tipo confirmatorio, utilizando como dimensiones latentes aquellos factores definidos por la propia teoría (Altemeyer, 1998). La Tabla 2 proporciona la información de los índices de ajuste del modelo que probamos y al modelo unifactorial.

Tabla 2. Indices de ajuste para los modelos factoriales

\begin{tabular}{|cccccc|}
\hline Estructura & $\mathrm{X}^{2}$ & CFI & NFI & RFI & RMSEA \\
\hline Un factor & $223.68^{*}$ & .97 & .97 & .95 & .09 \\
\hline $\begin{array}{c}\text { Tres } \\
\text { factores }\end{array}$ & $169.03^{*}$ & .98 & .97 & .96 & .08 \\
\hline
\end{tabular}

$$
{ }^{*} \mathrm{p}=.000
$$


En general, se puede apreciar que el ajuste es mejor en el modelo de tres factores en casi todos los índices obtenidos. Si bien los valores obtenidos para chi cuadrado nos indican que el modelo no se ajusta de forma perfecta, debemos recordar que éste es un índice muy sensible al tamaño de la muestra. En este sentido, los índices CFI (Comparative Fix Index), NFI (Normal Fit Index) y RFI (Relative Fix Index) muestran valores por encima de .90 , que son considerados como muy buenos (Bentler $\&$ Dudgeon, 1996). Eso sí, se puede apreciar que el modelo de tres factores obtiene índices más elevados que el de un solo factor. Este mejor ajuste se aprecia más claramente en el índice RMSEA (Root Mean Square Error of Approximation) que es significativo con valores inferiores a .08 (Browne $\&$ Kudeck, 1993).

Con el fin de estudiar la relación entre las variables medidas por las diferentes escalas utilizadas en el instrumento, hemos realizado un análisis de regresión múltiple (modelo estándar) de modo de testear el impacto de la SDO, y de las escalas de nacionalismo y patriotismo sobre la versión que estamos utilizando de la RWA. Tomadas en conjunto las tres variables independientes explican el $27 \%$ de la varianza de la variable dependiente, y la relación lineal entre las variables es significativa $\left(\mathrm{R}^{2}=.27 ; \mathrm{F}_{(3,259)}=\right.$ 32.16; $\mathrm{p}<.01)$. Cabe señalar que la variable que más peso tiene en la ecuación de regresión es la $\mathrm{SDO}(\beta=.27 ; \mathrm{p}<.01)$, seguida por la variable nacionalismo $(\beta=.26 ; \mathrm{p}<.01)$. La tercera de las variables independientes, patriotismo, es la que menos importancia adquiere, aunque, como las otras dos, también resulta significativa su asociación $(\beta=.19 ; \mathrm{p}<.01)$. Los resultados anteriores nos indicarían que el autoritarismo aumentaría en la medida en que lo hacen los niveles de dominancia social, nacionalismo y patriotismo.

Al analizar las correlaciones encontradas entre las diferentes dimensiones de la escala, apreciamos que todas ellas resultan moderadas, aunque significativas (ver Tabla 3). Las dimensiones de "Convencionalismo" y "Sumisión" obtienen la correlación más alta $(\mathrm{r}=.35)$, seguido por "Convencionalismo" y "Agresión" ( $r=.32)$ y finalmente entre "Sumisión" y "Agresión” ( $\mathrm{r}=.13)$. 
Tabla 3. Correlación entre dimensiones de la RWA

\begin{tabular}{|c|c|c|c|}
\hline & Convencionalismo & $\begin{array}{l}\text { Agresión } \\
\text { Autoritaria }\end{array}$ & $\begin{array}{l}\text { Sumisión } \\
\text { Autoritaria }\end{array}$ \\
\hline Convencionalismo & 1 & & \\
\hline Agresión Autoritaria & $.32^{* *}$ & 1 & \\
\hline Sumisión Autoritaria & $.35^{* *}$ & $.13^{*}$ & 1 \\
\hline
\end{tabular}

$\mathrm{Al}$ analizar las correlaciones obtenidas por las escalas incorporadas en el instrumento, observamos que éstas son positivas y significativas, aunque moderadas. De este modo, la escala RWA obtendría una correlación de .40 con la escala de Nacionalismo, de .37 con la SDO y de .33 con la de Patriotismo (todas significativas con $\mathrm{p}<.01$ ), lo que resulta coherente con las hipótesis que hemos planteado.

Para poner a prueba las restantes hipótesis hemos segmentado la muestra entre aquellas personas que se declaran no religiosas, aquellas medianamente religiosas (obtienen puntuaciones en la escala de religiosidad inferiores a la media de dicha escala para la muestra completa; $M=3.4$ y DT $=1.83$ ) y aquellas muy religiosas (puntuaciones superiores a la media de la escala). Las diferencias aparecen justamente entre los grupos extremos $\left(\mathrm{F}_{(2}\right.$, $\left.{ }_{261)}=2.92 ; \mathrm{p}=.05\right)$, es decir, entre las personas muy religiosas $(\mathrm{M}=5.22) \mathrm{y}$ las no religiosas $(M=4.92)$, siendo estas últimas significativamente menos autoritarias. Además, hemos podido constatar que dichas diferencias están radicadas en una de las tres dimensiones de la RWA, aquella que remite a la "Agresión Autoritaria" $\left.\mathrm{F}_{(2,261)}=8.34 ; \mathrm{p}<.01\right)$.

Posteriormente, hemos comparado a los sujetos dividiéndolos por sexo, pero no se han encontrado diferencias significativas en los niveles de autoritarismo de hombres $(\mathrm{M}=5.11$ y DT $=.82)$ y mujeres $(\mathrm{M}=5.06$ y DT $=.88)$. Es decir, no existirían diferencias entre sexos en la escala de autoritarismo de derechas. Tampoco se ha hallado diferencias significativas al segmentar la muestra por nivel socioeconómico. 
La que sí resulta una variable relevante es la auto-categorización política de los participantes $\left(\mathrm{F}_{(2,240)}=3.49 ; \mathrm{p}<.05\right)$, siendo los sujetos que se identifican con la derecha $(\mathrm{M}=5.51)$ significativamente más autoritarios que los que se identifican con la izquierda $(\mathrm{M}=4.91)$. Al extender esta comparación a las dimensiones de la escala, observamos, al igual que ocurrió en la escala de religiosidad, que las diferencias encontradas se ratifican sólo en la dimensión de "Agresión Autoritaria" de la RWA (F $(2,240)=6.12 ; \mathrm{p}<.01)$, lo que indicaría que las personas de derecha $(\mathrm{M}=$ 5.11) serían significativamente más proclives que aquellos de izquierda ( $M$ = 4.45) para avalar la agresión contra aquellas personas sancionadas por la autoridad "legítima".

La dimensión "Agresión Autoritaria” logra correlaciones más elevadas que las del conjunto de la RWA tanto con la SDO $(r=.43)$, con la medida de Nacionalismo $(\mathrm{r}=.41)$, la de Religiosidad $(.31)$ y con la de Patriotismo (.36). Todas estas correlaciones son significativas ( $\mathrm{p}<.01)$. Lo anterior nos indicaría que la dimensión que analizamos es un elemento diferenciador del autoritarismo de los participantes de nuestra muestra. $\mathrm{Al}$ realizar un análisis de regresión múltiple, utilizando la dimensión "Agresión Autoritaria" como variable dependiente, y las escalas SDO y de Nacionalismo como independientes, encontramos que el modelo logra explicar el $30 \%$ de la varianza, siendo la relación entre las variables significativa $\left(\mathrm{F}_{(2,260)}=65.63 ; \mathrm{p}<.01\right)$. La variable que más peso tiene en la ecuación de regresión es la SDO $(\beta=.49 ; \mathrm{p}<.01)$, y posteriormente, la variable nacionalismo $(\beta=.32 ; \mathrm{p}<.01)$.

\section{Discusión}

Los resultados obtenidos con la versión abreviada de la escala RWA nos revelan que se trataría de un instrumento fiable para medir autoritarismo de derechas en nuestro entorno social. La estructura factorial de la escala apunta a revelar la presencia de las tres dimensiones definidas por la teoría: "sumisión autoritaria", "agresión autoritaria" y "convencionalismo". Esta estructura factorial encuentra apoyo en los índices de ajuste 
obtenidos en la confirmación del modelo factorial. Sólo tres ítems de la escala parecen no funcionar de forma adecuada, obteniendo bajas correlaciones con el total, por lo que hemos decidido dejarlos fuera, dado que las tres dimensiones teóricas se mantuvieron representadas.

Por otra parte, hemos obtenido correlaciones significativas con otras escalas o medidas asociadas, tales como nacionalismo, religiosidad y dominancia. Eso sí, la medida referida a patriotismo también correlaciona significativamente con la RWA, a pesar que esperábamos que no lo hiciese. Lo anterior podría significar que los ítems de la escala RWA siguen compartiendo elementos con la escala SDO, sobre todo en aquellos ítems que se refieren a la relación con otras minorías sociales. En todo caso, dicha correlación es moderada (aunque significativa), lo que apuntaría a que serían fenómenos relacionados, pero distintos. En nuestra opinión, ambos contribuirían a identificar el estilo de pensamiento que podríamos denominar conservador. Por otra parte, la correlación encontrada entre la RWA y la medida de patriotismo puede ser explicada por el fuerte chovinismo expresado en alguno de sus ítems y en elementos contextuales, ya que en nuestra zona geográfica se aprecia un fuerte impulso hacia las estrategias de máxima diferenciación en la relación con las comunidades vecinas, dado que este territorio perteneció históricamente a los países limítrofes y que, de tanto en tanto, el tema es reflotado en la agenda política (Cárdenas, 2006).

Al comparar a los sujetos, utilizando como variable de segmentación sus niveles de religiosidad, nos encontramos con que los más religiosos son aquellos que más alto puntúan en la medida de autoritarismo de derechas. No se encontraron diferencias entre las personas que afirmaban no poseer creencias religiosas y aquellas que sí las tenían, pero que les asignaban una moderada importancia. Lo anterior nos indicaría que no es la presencia o ausencia de ideas religiosas, sino la importancia asignada a estas ideas en la vida cotidiana lo que relaciona con la medida de autoritarismo. Estas diferencias se hacen aún más llamativas cuando analizamos los resultados por dimensión, siendo la dimensión referida a "agresión autoritaria" la que mejor diferencia a los sujetos. Lo anterior nos podría estar indicando que mientras más importante es la religión para la vida de la persona, más 
tiende ésta a estructurarse sobre la idea de un orden y jerarquía natural, así como sobre la idea de existencia de una autoridad legítima y una ley que deriva de ella (ya sea ésta de orden natural o sobrenatural), siendo castigada cualquier violación a dicho orden "legítimo" o a dicha ley, y dirigiendo la agresión contra los perpetradores de dicha violación.

Por otra parte, nos encontramos también con diferencias significativas entre los sujetos al dividirlos utilizando como variable de segmentación sus ideas políticas. De este modo, las personas que más alto puntúan en la RWA son aquellas que se identifican con ideas de derecha, y aquellas que más bajo lo hacen son las que se autodefinen como de izquierda. Nuevamente encontramos que la dimensión que mejor diferencia a los sujetos es la tendencia a la "agresión autoritaria". Es decir, los sujetos más religiosos y aquellos con ideas de derecha son los que más de acuerdo están con las afirmaciones que apuntan a atacar o perjudicar a los miembros de grupos que atentan contra el poder establecido o en ocasiones en que la hostilidad dirigida hacia estos grupos es alentada por la autoridad.

En resumen, podemos concluir que la versión reducida de la escala RWA que hemos trabajado parece ser un instrumento fiable y válido para su uso en nuestro particular contexto, toda vez que aún cuenta con algunas limitaciones que deben ser subsanadas en estudios futuros. En particular, nos referimos al uso de muestras de conveniencia (incorporación de rangos etarios más amplios que permitan comparar grupos de edad diversos) y a la necesidad de intentar diferenciar aún más los ítems de la escala de aquellos contenidos referidos al deseo de dominancia respecto de otros grupos sociales. Por otra parte, en futuros estudios hay que reconsiderar las críticas que se han realizado a los instrumentos de auto-reporte de actitudes, y que obviamente esta escala comparte: diferente capacidad de las personas de darse cuenta o ser conscientes de sus propias actitudes y estados internos; la deseabilidad social de las respuestas de los sujetos (una persona que posee una actitud negativa hacia una persona o grupo no estará necesariamente dispuesta a revelarla, sobre todo en aquellos casos en que existen fuertes presiones sociales para asumir conductas tolerantes e igualitarias); a la presentación positiva que intentan hacer de sí mismos los 
sujetos o a los intentos de corregir sobre la marcha los juicios en aquellas escalas reactivas (Petty \& Wegener, 1998).

Finalmente, resulta relevante refinar los instrumentos que nos permiten acercarnos al estudio de los fenómenos políticos y psicosociales, dado que existe siempre la posibilidad de una reconfiguración de las condiciones que hacen posible el apego a sistemas de gobierno y formas de relación autoritarias, la sumisión ciega a dichas autoridades y la agresividad dirigida hacia las propias víctimas. La democracia no tiene umbrales que una vez atravesados aseguren su permanencia. Se trata más bien de un trabajo cotidiano, lento, siempre precario e incompleto. De allí la necesidad de mantenernos alertas y vigilantes, de allí la necesidad de contar con instrumentos cada vez más refinados para captar sus nueva formas de expresión y de estudiar bajo una nueva óptica un fenómeno psicosocial (en tanto articula dinámicas individuales y sociales) ya conocido. Este trabajo, no es sino una apuesta por adaptar uno de los instrumentos más conocidos sobre el tema, de modo de poner a disposición de los investigadores del área una versión revisada, breve pero fiable, y adaptada a nuestro contexto.

\section{Referencias}

Ahrens, C. R. \& Innes, J. M. (1994). Attribution of right-wing authoritarianism as a function of self-perceived political opinion. Journal of Social Psychology, 134, 383-385.

Altemeyer, B. (1981). Right-wing authoritarianism. Winnipeg: University of Manitoba Press.

Altemeyer, B. (1988). Enemies of freedom. San Francisco: Jossey-Bass.

Altemeyer, B. (1993). Nacionalismo y Autoritarismo de derechas entre legisladores americanos. Psicología Politica, 7, 7-18.

Altemeyer, B. (1996). The authoritarian specter. Cambridge MA: Harvard University Press.

Altemeyer, B. (1998). The other authoritarian personality. Advances in Experimental Social Psychology, 30, 47-92.

Bentler, M. P. \& Dudgeon, P. (1996). Covariance structure analysis: Statistical practice, theory and directions. Annual Review of Psychology, 47, 563-592. 
Browne, M. W. \& Kudeck, R. (1993). Alternative ways of assessing model fit. En K. A. Bollen \& J. S. Long (Eds.), Testing structural equation models (pp. 136-162). Newbury Park, CA: Sage.

Calderón, C. (2009). El Nacionalismo en la Era de la Globalización y su relación con el prejuicio étnico en una Muestra Representativa en las Ciudades de Antofagasta y Calama. Tesis de maestría no publicada. Antofagasta: Universidad Católica del Norte.

Cárdenas, M. (2006). "Y verás como quieren en Chile...": un estudio sobre prejuicio hacia los inmigrantes bolivianos por parte de jóvenes chilenos. Última Década, 24, 99-124.

Cárdenas, M., Meza, P., Lagues, K. \& Yáñez, S. (2010). Adaptación y Validación de la Escala de Orientación a la Dominancia Social (SDO) en una muestra chilena. Universitas Psychologica, 9(1), 165-172.

Cohrs, C. \& Abrock, F. (2009). Right-wing authoritarianism, social dominance orientation and prejudice against threatening and competitive ethnic groups. European Journal of Social Psychology, 39, 270-289.

Cohrs, J. C., Moschner, B., Maes, J. \& Kielmann, S. (2005). The motivational bases of right-wing authoritarianism and social dominance orientation: Relations to values and attitudes in the aftermath of September 11, 2001. Personality and Social Psychology Bulletin, 31, 1425-1434.

Dru, V. (2007). Authoritarianism, social dominance orientation and prejudice: Effects of various self-categorization conditions. Journal of Experimental Social Psychology, 43, 877-883.

Duckitt, J. \& Farre, B. (1994). Right wing authoritarianism and political intolerance among whites in the future majority-rule South Africa. Journal of Social Psychology, 134, 735-741.

Duckitt, J. (1992). The Social Psychology of Prejudice. New York: Praeger.

Duckitt, J. (2000). Culture, personality and prejudice. En S. A. Renshon \& J. Duckitt (Eds.) Political Psychology: cultural and cross-cultural foundations (pp. 89-107). New York: New York University Press.

Ekehammar, B., Akrami, N., Gylje, M. \& Zakrisson, I. (2004). What matters most to prejudice: Basic personality, social dominance orientation or right-wing authoritarianism? European Journal of Personality, 18, 463-482.

Farre, B. \& Duckitt, J. (1994). The validity of Tompkins's polarity scale among white South Africans. Journal of Social Psychology, 134, 287296.

Haddock, G., Zanna, M. P. \& Esses, V. M. (1993). Assesing the structure of prejudicial attitudes: the case attitudes of homosexuals. Journal of Personality and Social Psychology, 65, 1105-1118. 
Kellas, J.G. (1991). The Politics of Nationalism and Ethnicity. London: MacMillan.

Knight, K. (1999). Right-wing authoritarianism scale. En J. Robinson, P. Shaver \& L. Wrightsman (Eds.). Measures of Political Attitudes (pp. 59-158). San Diego: Academic Press.

Kosterman, R. \& Feshbach, S. (1989). Towards a measure of patriotic and nationalistic attitude. Political Psychology, 10, 257-274.

McFarland, S. G., Agayev, V. S. \& Abalakina, M. A. (1992). Authoritarianism in the Former Soviet Union. Journal of Personality and Social Psychology, 63 (6), 1004-1010.

McFarland, S. G., Ageyev, V. S. \& Djintcharadze, N. (1996). Russian authoritarianism two years after communism. Personality and Social Psychology Bulletin, 22, 210-217.

Meloen, J., VanderLinden, G. \& De Witte, H. (1996). A test of approaches of Adorno et al., Lederer and Altemeyer of authoritarianism in Belgian Flanders: a research note. Political Psychology, 17, 643-656.

Petty, R. E., \& Wegener, D. T. (1998). Attitude change: Multiple roles for persuasion variables. En D. Gilbert, S. Fiske \& G. Lindzey (Eds.). The handbook of social psychology (4a ed., pp. 323-390). New York: McGraw-Hill.

Pratto, F., Sidanius, J., Stallworth, L. \& Malle, B. (1994). Social Dominance Orientation: A personality variable predicting social and political attitudes. Journal of Personality and Social Psychology, 67, 741-763.

Ray, J. J. (1985). The punitive personality. Journal of Social Psychology, 25, 329-334.

Roccato, M. (1997). Autoritarismo de derechas y adolescencia. Psicología Politica, 14, 61-76.

Roccato, M., Re, M. \& Sclauzero, S. (2002). Autoritarismo, Nacionalismo y Patriotismo. Un estudio con militantes italianos. Psicología Política, 25, 21-35.

Rosenberg, P. (2006). Right-wing Authoritarianism and Conservative Identity Politics. (Recuperado el 10 de mayo de 2010) de: http://patternsthatconnect.blogspot.com/2006/03/rightwingauthoritarianism-and.html

Schaffer, B. S. \& Riordan, C. M. (2003). A review of cross-cultural methodologies for organizational research: A best practices approach. Organizational Research Methods, 6, 169-215.

Schultz, P. W. \& Stone, W. F. (1994). Environmental attitudes and authoritarianism: field and laboratory perspectives. Environment and Behavior, 26, 25-37.

Seoane, J. \& Garzón, A. (1992). Creencias sociales contemporáneas, autoritarismo y humanismo. Psicología Politica, 5, 27-52. 
Sidanius, J. \& Pratto, F. (1999). Social Dominance: An intergroup theory of social hierarchy and oppression. Cambridge, UK: Cambridge University Press.

Stone, W. \& Schaffner, P. E. (1997). The Tomkins Polarity Scale: Recent Developments. Bulletin of the Tomkins Institute, 4, 17-22.

Stones, C. R. (2006). Anti-gay attitudes among heterosexual males: rightwing authoritarianism as a stronger predictor than social-dominance orientation and heterosexual identity. Social behavior and personality, 34(9), 1137-1150.

Therune, K. W. (1964). Nationalism among foreign and American students: an exploratory study. The Journal of Conflict Resolution, 8(3), 256-270.

Thomsen, L., Green, E. \& Sidanius, J. (2008). We will hunt them down: How social dominance orientation and right-wing authoritarianism fuel ethnic persecution of immigrants in fundamentally different ways. Journal of Experimental Social Psychology, 44, 1455-1464.

Walter, M. I. (1996). Personality and Emotion: Response to musical mood induction. Tesis de maestría no publicada, University of Maine, Orono, EE.UU.

Zakrisson, I. (2005). Construction of a short version of Right-wing Authoritarianism (RWA) scale. Personality and Individual Differences, 39, 863-872.

Zwillenberg, D. F. (1983). Predicting biases in the punishment of criminals as a function of authoritarianism: Effects of severity of the crime, degree of mitigating circumstances, and status of the offender. Disertación doctoral no publicada, Columbia University, New York, EE.UU.

Fecha de recepción: 20 de abril de 2010.

Fecha de aceptación: 27 de julio de 2010. 


\section{Apéndice}

\section{Escala RWA - versión de 12 ítems}

1.- Nuestra sociedad necesita líderes fuertes que puedan erradicar el extremismo y la inmoralidad que prevalecen actualmente.

2.- Nuestro sociedad necesita libres pensadores, que tengan la valentía para confrontar los convencionalismos, incluso si esto molestase a muchas personas.

3.- Las tradiciones y valores antiguos aún nos indican la mejor forma de vivir.

4.- Nuestra sociedad sería mejor si mostráramos tolerancia y comprensión por las ideas y valores diferentes (no convencionales).

5.- Las leyes que castigan el aborto y la pornografía, y que contribuyen a proteger el matrimonio deben ser estrictamente acatadas. Las transgresiones deben castigarse severamente.

6.- La sociedad necesita mostrar una mayor apertura hacia las personas que piensan por sí mismas y diferente de las autoridades, más que apoyar el que dichas autoridades decidan por nosotros.

7.- Muchas personas desafían al Estado, critican a la Iglesia e ignoran las formas normales de vida, sin que por ello dejen de ser buenas.

8.- Nuestros antepasados deben ser más admirados y respetados por su contribución a la construcción de nuestra sociedad, sobre todo en esta época en que existen fuerzas que tratan de destruirla.

9.- Hay muchas personas radicales o inmorales que tratan de echar a perder las cosas; la sociedad debe frenarlos.

10.- En defensa de la libertad de expresión deberíamos permitir la publicación de literatura que incluso podemos considerar mala o contraria a nuestras ideas.

11.- La situación de la sociedad actual mejoraría si los agitadores fueran tratados con humanidad e intentando hacerlos entrar en razón.

12.- Todo buen ciudadano debería ayudar a eliminar la maldad que envenena nuestro país desde dentro si la sociedad así lo requiere. 